Archive for

Organic Chemistry

Arkivoc 2017, part iv, 236-248

\title{
A facile base-promoted domino Michael/O-alkylation reaction for the construction of succinimide-substituted $3(2 H)$-furanones
}

\author{
Wu Wang, Guojuan Liang, Yan Bai, Lijuan Bai, Hui Zhou, Yu Yu, and Jing Zhou* \\ School of Pharmaceutical Science, Chongqing Research Center for Pharmaceutical Engineering, \\ Chongqing Key Laboratory of Biochemistry and Molecular Pharmacology, Chongqing Medical University, \\ Chongqing 400016, China \\ Email: zhoujing045@cqmu.edu.cn
}

Received 01-11-2017

Accepted 03-13-2017

Published on line 05-29-2017

\section{Abstract}

A novel base-promoted domino Michael/O-alkylation reaction of maleimides with $\gamma$-halogenated- $\beta$-ketoesters is described. A variety of new succinimide-substituted $3(2 \mathrm{H})$-furanones were obtained in excellent yields (up to $96 \%)$ under simple and mild conditions. The structure of the new compound 3a was determined by singlecrystal X-ray analysis and a reaction pathway is proposed.<smiles>[R]N1C(=O)C=CC1=O</smiles><smiles>[R]CC(=O)CC(=O)O[Tl]</smiles>

$\mathrm{R}^{2}=\mathrm{Cl}, \mathrm{Br}$

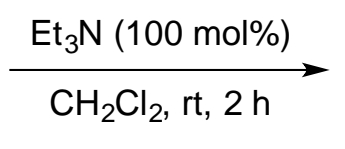<smiles>[R9]OC1=C([C@H]2CC(=O)N([R7])C2=O)C(=O)CO1</smiles>

16 examples up to $96 \%$ yield

Keywords: Succinimide-substituted 3(2H)-furanones, Michael/O-alkylation, domino reaction, maleimides, $\nu$-halogenated- $\beta$-ketoesters 


\section{Introduction}

$3(2 H)$-Furanones are core structural motifs that are widely present in many natural products and pharmaceutically important compounds. Substituted $3(2 \mathrm{H})$-furanones show a wide range of biological activities such as anti-inflammatory, antiallergic, antitumor and anti-ulcer activities (examples in Figure 1). ${ }^{1-4}$ The significance of these molecules has led to a variety of approaches for the synthesis of substituted $3(2 \mathrm{H})$ furanones, including acid-catalyzed cyclization-dehydration, ${ }^{5,6}$ transformations from furans, ${ }^{7,8}$ alkynes ${ }^{9-15}$ and allenes. ${ }^{16}$ However, most of these routes require the use of specific substrates and reaction conditions are often harsh. Domino reactions are one of the most efficient strategies for the synthesis of complex molecular structures from simple materials in a single step. ${ }^{17-20}$ Recently, $\gamma$-halogenated- $\beta$-ketoesters, which are commercially available, were used to construct substituted $3(2 H)$-furanones via domino reactions with activated alkenes ${ }^{21-23}$ and imines. ${ }^{24-26}$ This synthetic strategy is efficient and mild. However the activated alkenes were limited only to chain structures such as chain nitroalkenes ${ }^{21,22}$ and chain $\alpha, \beta$-unsaturated esters. ${ }^{23}$ Thus it was important to discover whether other diverse activated alkenes, such as cyclic alkenes, would react with $\gamma$-halogenated- $\beta$-ketoesters to construct diverse substituted $3(2 H)$-furanones.

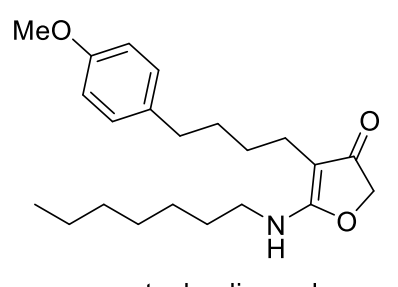

aza-prostaglandin analogue

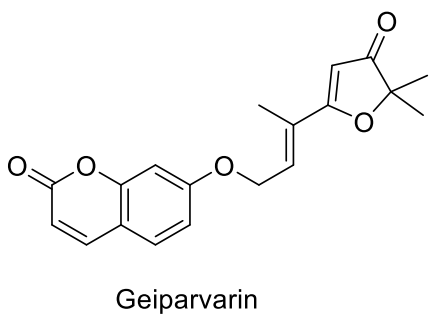

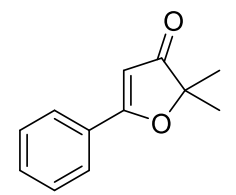

Bullatenone

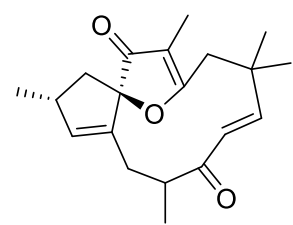

Jatrophone

Figure 1. Bioactive products with a $3(2 H)$-furanone subunit.

Maleimides are an important class of activated cyclic alkenes. They have been extensively applied in organic synthesis to construct substituted succinimides and functionalized pyrrolidines, which are core structural units found in natural products and clinical drug candidates. ${ }^{27-31}$ To date, there has been no report

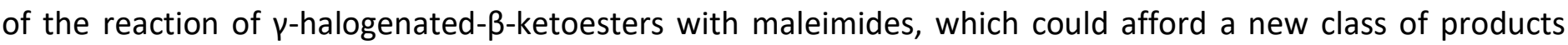
combining a biologically significant succinimide with a $3(2 H)$-furanone. These fused products might show higher or new biological activities. As a part of our continuing interest in the construction of complex and novel drug candidates, ${ }^{32-36}$ herein, we report the first domino Michael/O-alkylation reaction of $\gamma$-halogenated$\beta$-ketoesters with maleimides to access a new range of succinimide-substituted $3(2 \mathrm{H})$-furanones. According to the literature, $\gamma$-halogenated- $\beta$-ketoesters react with $\alpha, \beta$-unsaturated aldehydes to afford cyclopentanone products via Michael/ $\alpha$-alkylation. ${ }^{37}$ These reports show variable chemical reactivities of $\gamma$-halogenated- $\beta$ - 
ketoesters with activated alkenes. Our preliminary studies involved maleimide 1a and ethyl 4-chloroacetoacetate $\mathbf{2 a}$ as substrates, these were allowed to react in dichloromethane at room temperature in the presence of 100 mol\% $\mathrm{Et}_{3} \mathrm{~N}$. The reaction worked well and gave succinimide-substituted $3(2 \mathrm{H})$-furanone $3 a$ via a domino Michael/O-alkylation process - not a Michael/ $\alpha$-alkylation process (Scheme 1 ).

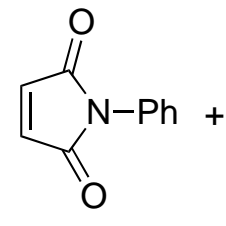

$1 \mathrm{a}$

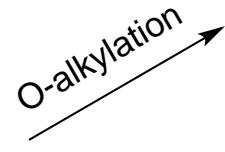

$\mathrm{Et}_{3} \mathrm{~N}$

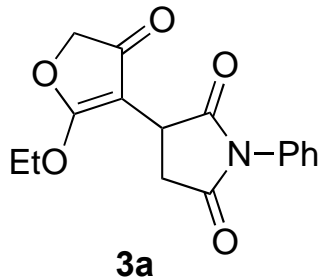

$3 a$

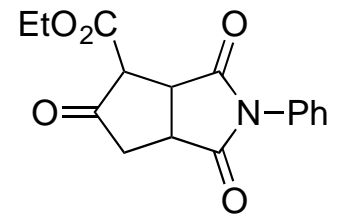

succinimide-substituted $3(2 H)$-furanone

domino Michael/O-alkylation reaction

Scheme 1. Domino Michael/O-alkylation reaction of $1 \mathbf{a}$ and $\mathbf{2 a}$.

\section{Results and Discussion}

To evaluate the role of the base in this system, the reaction of $\mathrm{N}$-phenylmaleimide 1a with ethyl 4-chloroacetoacetate $\mathbf{2 a}$ was used as a model reaction, and a series of bases were investigated in dichloromethane at room temperature, and the results were shown in Table 1. Weak bases such as $\mathrm{Na}_{2} \mathrm{CO}_{3}$ and $\mathrm{NaHCO}_{3}$ decreased the reaction rate and afforded poor yields after reaction for $24 \mathrm{~h}$ (Table 1, entries 2 and 3). Strong base DABCO also afforded a poor yield because impurities were produced (Table 1, entry 5 ). $\mathrm{Et}_{3} \mathrm{~N}$ gave the highest yield and was chosen as the most suitable base ( $92 \%$ yield, Table 1 , entry 1$)$. Next, the reaction was conducted in various solvents (Table 1, entries 7-14). Among them, $\mathrm{MeOH}$ afforded a poor yield because impurities were produced (Table 1, entry 13), $n$-hexane afforded only a trace of product because of poor solubility (Table 1 , entry 14). Other solvents afforded $3 a$ in moderate to good yields (80-90\% yield, Table 1, entries 7-12). Dichloromethane gave the highest yield and was selected as the most suitable reaction media for further optimization (Table 1, entry 1).

Increasing or decreasing the amount of $\mathrm{Et}_{3} \mathrm{~N}$ gave a lower yield (Table 2, entries 2-4 vs. 1) so having identified 100 mol\% $\mathrm{Et}_{3} \mathrm{~N}$ as the optimal loading for the reaction, we next examined the effect of the reaction temperature (Table 2, entries 5 and 6). A screening of different reaction temperatures showed that the reaction gave the best results at room temperature (Table 2, entry 1). Decreasing reaction temperature slowed down the reaction rate thus decreased the yield (Table 2, entry 5). Increasing the reaction temperature also decreased the yield because impurities were produced (Table 2, entry 6). Finally, the substrate concentration was examined (Table 2, entries 7 and 8). It was found that increasing the substrate concentration slightly decreased the yield (Table 2, entry 7), lowering the substrate concentration decreased the reaction rate and gave lower yield ( $82 \%$ yield, Table 2, entry 8 ), $0.2 \mathrm{M}$ was the optimal substrate 
concentration. Consequently, the following reaction conditions are recommended: 100 mol\% $\mathrm{Et}_{3} \mathrm{~N}$ with $0.2 \mathrm{M}$ substrate in $\mathrm{CH}_{2} \mathrm{Cl}_{2}$ at room temperature (Table 2, entry 1 ).

Table 1. Optimization of reaction conditions ${ }^{a}$

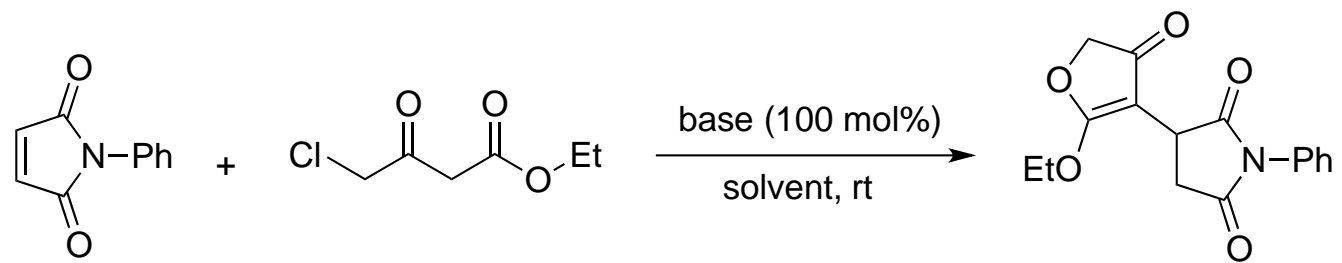

\begin{tabular}{ccccc}
\multicolumn{1}{c}{$\mathbf{2 a}$} & \multicolumn{3}{c}{ 3a } \\
\hline Entry & Solvent & Base & Time/h & Yield $^{b} \%$ \\
\hline 1 & $\mathrm{CH}_{2} \mathrm{Cl}_{2}$ & $\mathrm{Et}_{3} \mathrm{~N}$ & 2 & 92 \\
2 & $\mathrm{CH}_{2} \mathrm{Cl}_{2}$ & $\mathrm{NaHCO}_{3}$ & 24 & trace \\
3 & $\mathrm{CH}_{2} \mathrm{Cl}_{2}$ & $\mathrm{Na}_{2} \mathrm{CO}_{3}$ & 24 & 56 \\
4 & $\mathrm{CH}_{2} \mathrm{Cl}_{2}$ & $\mathrm{NaOH}$ & 2 & 88 \\
5 & $\mathrm{CH}_{2} \mathrm{Cl}_{2}$ & $\mathrm{DABCO}$ & 2 & 21 \\
6 & $\mathrm{CH}_{2} \mathrm{Cl}_{2}$ & $\mathrm{DBU}$ & 2 & 90 \\
7 & $\mathrm{Et}_{2} \mathrm{O}$ & $\mathrm{Et}_{3} \mathrm{~N}$ & 2 & 80 \\
8 & $\mathrm{THF}$ & $\mathrm{Et}_{3} \mathrm{~N}$ & 2 & 85 \\
9 & $\mathrm{toluene}_{10}$ & $\mathrm{Et}_{3} \mathrm{~N}$ & 2 & 82 \\
11 & $\mathrm{EtOAC}$ & $\mathrm{Et}_{3} \mathrm{~N}$ & 2 & 80 \\
12 & $\mathrm{CH}{ }_{3} \mathrm{CN}$ & $\mathrm{Et}_{3} \mathrm{~N}$ & 2 & 90 \\
13 & $\mathrm{DMF}$ & $\mathrm{Et}_{3} \mathrm{~N}$ & 2 & 87 \\
14 & $\mathrm{Em}$ & 2 & 18 \\
\hline
\end{tabular}

a Unless otherwise noted, reactions were conducted with $0.2 \mathrm{mmol} 1 \mathrm{a}, 0.2 \mathrm{mmol} 2 \mathrm{a}, 100 \mathrm{~mol} \%$ base, in $1.0 \mathrm{~mL}$ solvent at room temperature. ${ }^{b}$ Isolated yields. 
Table 2. Optimization of reaction conditions ${ }^{a}$<smiles>O=C1C=CC(=O)N1[PH3+]</smiles>

1a<smiles>CCOC(=O)CC(=O)CCl</smiles>

2a<smiles>CCN(CC)CC</smiles>

EtO<smiles>CCOC1=C(C2CC(=O)N(c3ccccc3)C2=O)C(=O)CO1</smiles>

3a

\begin{tabular}{cccc}
\hline Entry & $\mathrm{x}$ & $T /{ }^{\circ} \mathrm{C}$ & Yield $^{b} / \%$ \\
\hline 1 & 100 & 25 & 92 \\
2 & 200 & 25 & 92 \\
3 & 300 & 25 & 90 \\
4 & 50 & 25 & 69 \\
5 & 100 & 0 & 70 \\
6 & 100 & 40 & 91 \\
$7^{c}$ & 100 & 25 & 89 \\
$8^{d}$ & 100 & 25 & 82 \\
\hline
\end{tabular}

${ }^{a}$ Unless otherwise noted, reactions were conducted with $0.2 \mathrm{mmol} 1 \mathrm{a}, 0.2 \mathrm{mmol} 2 \mathrm{a}, \mathrm{x}$ mol\% base, in $1.0 \mathrm{~mL}$ $\mathrm{CH}_{2} \mathrm{Cl}_{2}$. ${ }^{b}$ Isolated yields. ${ }^{c} 0.5 \mathrm{~mL} \mathrm{CH} \mathrm{Cl}_{2}$ was used. ${ }^{d} 2.0 \mathrm{~mL} \mathrm{CH} \mathrm{Cl}_{2}$ was used.

Under the optimal reaction conditions, the generality of this protocol was studied (Table 3). Firstly, a wide range of maleimides 1a-n was studied (Table 3, entries 1-14). The maleimides included those bearing electronwithdrawing and electron-donating substituents on the aryl ring, as well as $N$-alkyl maleimides. All gave good yields (85-96\%). The electronic properties and position of the substituents on the $N$-aryl maleimides phenyl ring affected the yield slightly (Table 3 , entries 1-9). The $N$-aryl maleimide with a strong electron-withdrawing nitro group, 1g, gave a slightly lower yield (Table 3, entry 7). Maleimide $1 \mathrm{~m}$ also gave a slightly lower yield due to the larger steric hindrance (Table 3, entry 13 ). In addition, methyl 4-chloroacetoacetate $\mathbf{2 b}$ and ethyl 4bromoacetoacetate 2c were also tested, both provided excellent yields (Table 3, entries 15 and 16). The structure of 3a was determined by an X-ray analysis of a single crystal (Figure 2). ${ }^{38}$ 
Table 3. Scope of substrates ${ }^{a}$

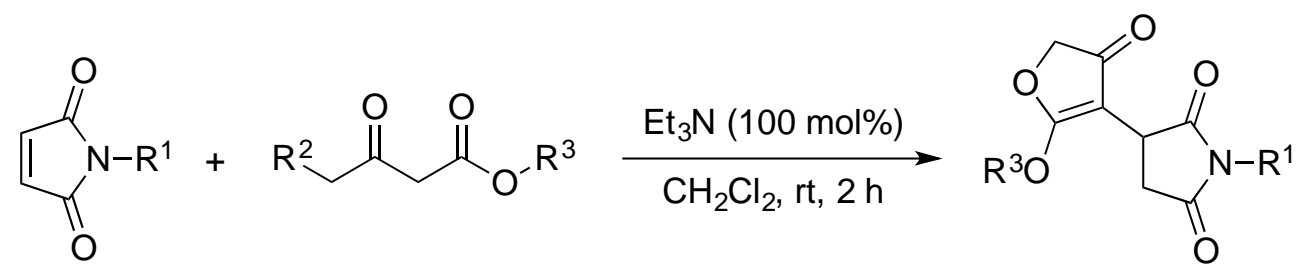

\begin{tabular}{|c|c|c|c|c|}
\hline Entry & $1 / R^{1}$ & $2 / R^{2}, R^{3}$ & 3 & Yield $^{b} / \%$ \\
\hline 1 & $1 \mathrm{a} / \mathrm{C}_{6} \mathrm{H}_{5}$ & $2 \mathrm{a} / \mathrm{Cl}, \mathrm{Et}$ & $3 a$ & 92 \\
\hline 2 & $\mathbf{1 b} / 4-\mathrm{CH}_{3} \mathrm{C}_{6} \mathrm{H}_{4}$ & $2 \mathrm{a} / \mathrm{Cl}, \mathrm{Et}$ & $3 b$ & 95 \\
\hline 3 & $1 \mathrm{c} / 4-\mathrm{CH}_{3} \mathrm{OC}_{6} \mathrm{H}_{4}$ & $2 \mathrm{a} / \mathrm{Cl}, \mathrm{Et}$ & $3 c$ & 93 \\
\hline 4 & $1 \mathrm{~d} / 4-\mathrm{FC}_{6} \mathrm{H}_{4}$ & $2 \mathrm{a} / \mathrm{Cl}, \mathrm{Et}$ & $3 d$ & 95 \\
\hline 5 & $1 \mathrm{e} / 4-\mathrm{ClC}_{6} \mathrm{H}_{4}$ & $2 \mathrm{a} / \mathrm{Cl}, \mathrm{Et}$ & $3 e$ & 93 \\
\hline 6 & $\mathbf{1 f} / 4-\mathrm{BrC}_{6} \mathrm{H}_{4}$ & $2 a / C l, E t$ & $3 f$ & 92 \\
\hline 7 & $1 \mathrm{~g} / 3-\mathrm{NO}_{2} \mathrm{C}_{6} \mathrm{H}_{4}$ & $2 a / C l, E t$ & $3 g$ & 85 \\
\hline 8 & $1 \mathrm{~h} / 3-\mathrm{FC}_{6} \mathrm{H}_{4}$ & $2 a / C l, E t$ & $3 h$ & 87 \\
\hline 9 & $1 \mathrm{i} / 2-\mathrm{MeC}_{6} \mathrm{H}_{4}$ & $2 a / C l, E t$ & $3 \mathbf{i}$ & 91 \\
\hline 10 & $1 \mathrm{j} / \mathrm{CH}_{3}$ & $2 a / C l, E t$ & $3 \mathbf{j}$ & 96 \\
\hline 11 & 1k/cyclohexyl & $2 a / C l, E t$ & $3 \mathbf{k}$ & 92 \\
\hline 12 & $1 \mathrm{l} / \mathrm{Bn}$ & $2 \mathrm{a} / \mathrm{Cl}, \mathrm{Et}$ & 31 & 90 \\
\hline 13 & $1 \mathrm{~m} / \mathrm{CHPh}_{2}$ & $2 a / C l, E t$ & $3 m$ & 88 \\
\hline 14 & 1n/1-naphthyl & $2 a / C l, E t$ & $3 n$ & 92 \\
\hline 15 & $1 \mathrm{a} / \mathrm{C}_{6} \mathrm{H}_{5}$ & 2b/Cl, Me & 30 & 94 \\
\hline 16 & $1 \mathrm{a} / \mathrm{C}_{6} \mathrm{H}_{5}$ & $2 \mathrm{c} / \mathrm{Br}, \mathrm{Et}$ & $3 a$ & 96 \\
\hline
\end{tabular}

${ }^{a}$ Unless otherwise noted, reactions were conducted with $0.2 \mathrm{mmol} 1,0.2 \mathrm{mmol} 2,100 \mathrm{~mol} \% \mathrm{Et}_{3} \mathrm{~N}$, in $1.0 \mathrm{~mL}$ $\mathrm{CH}_{2} \mathrm{Cl}_{2}$ at room temperature. ${ }^{b}$ Isolated yield. 


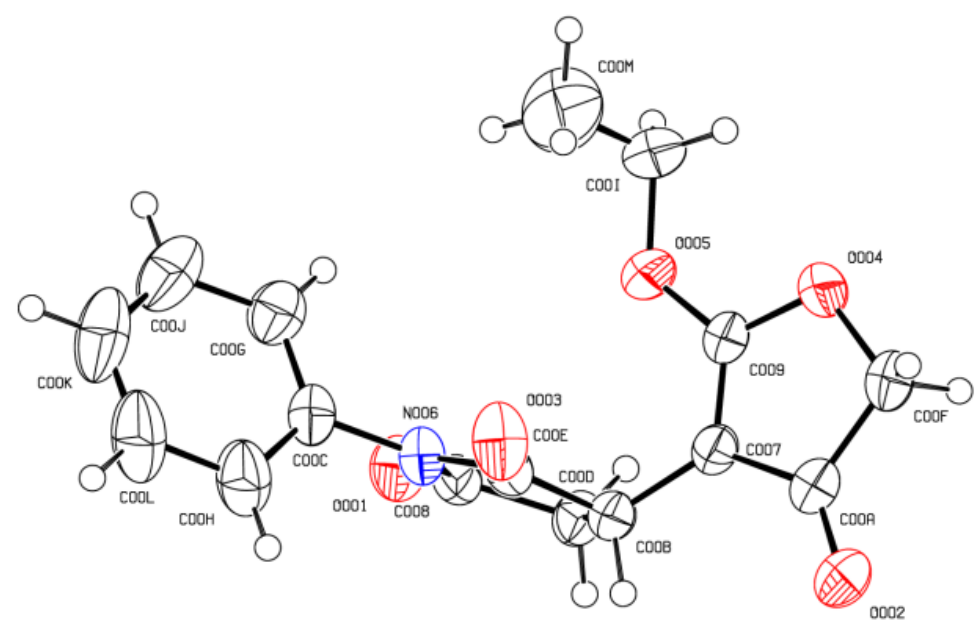

Figure 2. ORTEP representation of the X-ray crystal structure of product 3a (thermal ellipsoids set to $30 \%$ probability level).

Based on the experimental results and the observed structure of 3a, a proposed reaction pathway for the base-promoted domino Michael/O-alkylation reaction of maleimides $\mathbf{1}$ and $\boldsymbol{\gamma}$-halogenated- $\beta$-ketoesters $\mathbf{2}$ is shown in Scheme $2 .{ }^{23}$ First, the base deprotonates the $\beta$-ketoester to form an enolate, then nucleophilic conjugate addition to the maleimides by the enolate generates an intermediate Michael adduct $\mathbf{A}$. Next, in the presence of base, adduct $\mathbf{A}$ forms an enolate which undergoes an intramolecular O-alkylation process to form the product 3.
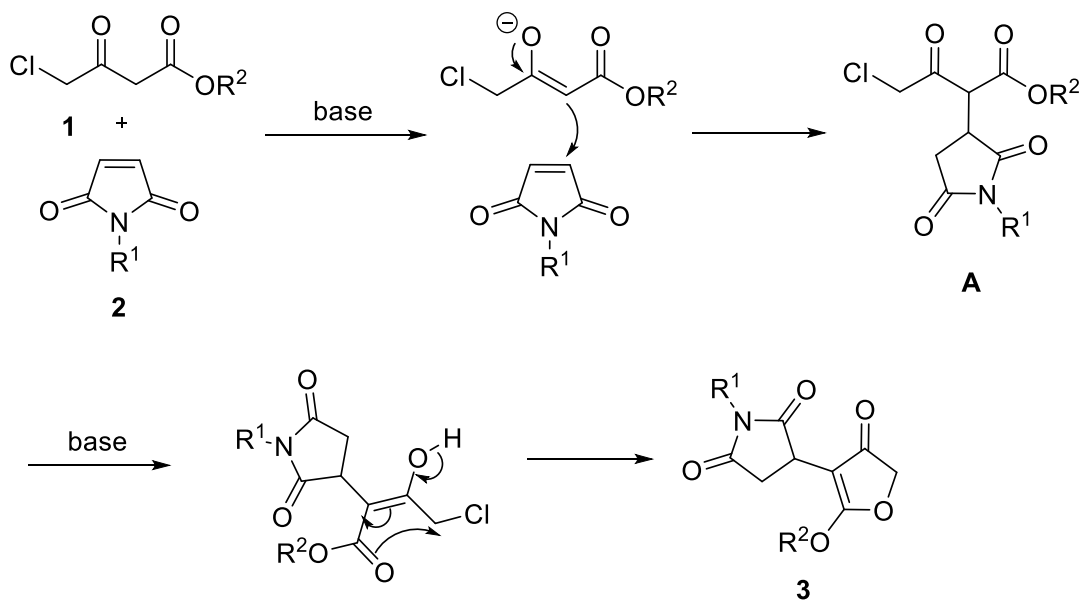

Scheme 2. Proposed pathway for the base-promoted domino Michael/O-alkylation reaction.

\section{Conclusions}

We have demonstrated a facile base-promoted domino Michael/O-alkylation reaction of maleimides and $\gamma$ halogenated- $\beta$-ketoesters. The reaction conditions are simple and mild. With this protocol, a wide range of 
new succinimide-substituted $3(2 H)$-furanones were smoothly obtained in good yields (up to $96 \%$ ). Further, expansion of these new succinimide-substituted $3(2 \mathrm{H})$-furanones to access products with known biological activities or new biologically significant molecules and testing their pharmacological activities are ongoing in our laboratory.

\section{Experimental Section}

General. $N$-Substituted maleimides 1 were prepared according to the literature method or similarly. ${ }^{39} \mathrm{Y}^{-}$ Halogenated- $\beta$-ketoesters $\mathbf{2}$ were purchased from commercial suppliers and used without further purification. Commercial grade solvents were dried and purified by standard procedures as specified in Purification of Laboratory Chemicals, 4th Ed (Armarego, W. L. F.; Perrin, D. D. Butterworth Heinemann: 1997). All melting points were measured on a SGWX-4 micro melting point apparatus. ${ }^{1} \mathrm{H}$ NMR spectra were recorded at $600 \mathrm{MHz}$. Chemical shifts were reported in ppm from tetramethylsilane with the solvent resonance as the internal standard $\left(\mathrm{CDCl}_{3}, \delta=7.26\right)$. Spectra are reported as follows: chemical shift $(\delta \mathrm{ppm})$, multiplicity $(\mathrm{s}=\mathrm{singlet}, \mathrm{d}=$ doublet, $\mathrm{t}=$ triplet, $\mathrm{q}=$ quartet, $\mathrm{m}=$ multiplet), coupling constants $(\mathrm{Hz})$, integration, and assignment. ${ }^{13} \mathrm{C} \mathrm{NMR}$ spectra were collected at $150 \mathrm{MHz}$ with complete proton decoupling. Chemical shifts are reported in ppm from the tetramethylsilane with the solvent resonance as internal standard $\left(\mathrm{CDCl}_{3}, \delta=77.0\right)$. Mass spectra were recorded on Bruker micrOTOF-Q II mass spectrometer. Reactions were monitored by TLC and visualized with ultraviolet light.

General procedure for the domino Michael/O-alkylation reaction. A solution of maleimide $1(0.20 \mathrm{mmol}, 1$ equiv), $\gamma$-halogenated- $\beta$-ketoester $2\left(0.20 \mathrm{mmol}, 1\right.$ equiv) in $\mathrm{CH}_{2} \mathrm{Cl}_{2}(1.0 \mathrm{~mL})$ was stirred at rt and $\mathrm{Et}_{3} \mathrm{~N}(0.20$ $\mathrm{mmol}, 100 \mathrm{~mol} \%)$ was added at the same temperature. The reaction mixture was stirred at $\mathrm{rt}$ for $2 \mathrm{~h}$ and concentrated in vacuo. The crude product was purified by column chromatography on silica gel (eluent $P E: E t O A c=2: 1)$ to afford pure products 3.

3-(2-Ethoxy-4-oxo-4,5-dihydrofuran-3-yl)-1-phenylpyrrolidine-2,5-dione (3a). White solid; 92\% yield; mp 112.5-113.5 ${ }^{\circ} \mathrm{C} .{ }^{1} \mathrm{H} \mathrm{NMR}\left(\mathrm{CDCl}_{3}, 600 \mathrm{MHz}\right) \delta 1.32(\mathrm{t}, J 7.14 \mathrm{~Hz}, 3 \mathrm{H}), 2.82$ (dd, J 5.58, $\left.18.18 \mathrm{~Hz}, 1 \mathrm{H}\right), 3.01(\mathrm{dd}, J$ 9.84, $18.18 \mathrm{~Hz}, 1 \mathrm{H}), 3.74-3.77(\mathrm{~m}, 1 \mathrm{H}), 4.37-4.40(\mathrm{~m}, 2 \mathrm{H}), 4.53(\mathrm{~s}, 2 \mathrm{H}), 7.23(\mathrm{~d}, J 7.50 \mathrm{~Hz}, 2 \mathrm{H}), 7.30(\mathrm{t}, J 7.50 \mathrm{~Hz}$, 1H), 7.38 (t, J $7.80 \mathrm{~Hz}, 2 \mathrm{H}) ;{ }^{13} \mathrm{C} \mathrm{NMR}\left(\mathrm{CDCl}_{3}, 150 \mathrm{MHz}\right) \delta$ 14.6, 33.2, 34.4, 66.4, 75.2, 90.8, 126.6, 128.5, 129.0, 132.3, 175.3, 176.3, 181.1, 194.1; HRMS (ESI) Calcd. for $\mathrm{C}_{16} \mathrm{H}_{15} \mathrm{NNaO}_{5}[\mathrm{M}+\mathrm{Na}]^{+}$: 324.0848; Found: 324.0842. 38. See supporting information file 2 for crystallographic data; the CCDC number is 1476572.

3-(2-Ethoxy-4-oxo-4,5-dihydrofuran-3-yl)-1-(p-tolyl)pyrrolidine-2,5-dione (3b). White solid; 95\% yield; mp 161.2-162.2 ${ }^{\circ} \mathrm{C} .{ }^{1} \mathrm{H} \mathrm{NMR}\left(\mathrm{CDCl}_{3}, 600 \mathrm{MHz}\right) \delta 1.31(\mathrm{t}, J 7.14 \mathrm{~Hz}, 3 \mathrm{H}), 2.28(\mathrm{~s}, 3 \mathrm{H}), 2.80(\mathrm{dd}, J 5.52,18.12 \mathrm{~Hz}, 1 \mathrm{H})$, $2.99(\mathrm{dd}, J 9.84,18.12 \mathrm{~Hz}, 1 \mathrm{H}), 3.72-3.75(\mathrm{~m}, 1 \mathrm{H}), 4.35-4.39(\mathrm{~m}, 2 \mathrm{H}), 4.52(\mathrm{~s}, 2 \mathrm{H}), 7.10(\mathrm{~d}, J 8.28 \mathrm{~Hz}, 2 \mathrm{H}), 7.17(\mathrm{~d}$,

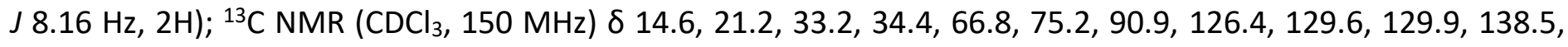
175.4, 176.4, 181.1, 194.0; HRMS (ESI) Calcd. for $\mathrm{C}_{17} \mathrm{H}_{17} \mathrm{NNaO}_{5}[\mathrm{M}+\mathrm{Na}]^{+}$: 338.1004; Found: 338.0999.

3-(2-Ethoxy-4-oxo-4,5-dihydrofuran-3-yl)-1-(4-methoxyphenyl)pyrrolidine-2,5-dione (3c). White solid; 93\% yield; mp 161.5-162.8 ${ }^{\circ} \mathrm{C} .{ }^{1} \mathrm{H}$ NMR $\left(\mathrm{CDCl}_{3}, 600 \mathrm{MHz}\right) \delta 1.32(\mathrm{t}, J 7.08 \mathrm{~Hz}, 3 \mathrm{H}), 2.78(\mathrm{dd}, J 5.46,18.12 \mathrm{~Hz}, 1 \mathrm{H})$, $2.98(\mathrm{dd}, J$ 9.84, $18.18 \mathrm{~Hz}, 1 \mathrm{H}), 3.71(\mathrm{~m}, 4 \mathrm{H}), 4.36-4.40(\mathrm{~m}, 2 \mathrm{H}), 4.52(\mathrm{~s}, 2 \mathrm{H}), 6.88(\mathrm{~d}, J 8.76 \mathrm{~Hz}, 2 \mathrm{H}), 7.13(\mathrm{~d}, J$ 8.76 Hz, 2H); ${ }^{13} \mathrm{C} \mathrm{NMR}\left(\mathrm{CDCl}_{3}, 150 \mathrm{MHz}\right) \delta$ 13.6, 32.1, 33.3, 54.4, 65.8, 74.2, 89.9, 113.3, 123.8, 126.6, 126.8, 158.4, 174.5, 180.1, 193.1; HRMS (ESI) Calcd. for $\mathrm{C}_{17} \mathrm{H}_{17} \mathrm{NNaO}_{5}[\mathrm{M}+\mathrm{Na}]^{+}$: 354.0954; Found: 354.0948. 
3-(2-Ethoxy-4-oxo-4,5-dihydrofuran-3-yl)-1-(4-fluorophenyl)pyrrolidine-2,5-dione (3d). White solid; 95\% yield; mp 192.0-193.0 ${ }^{\circ}$ C. $^{1} \mathrm{H}$ NMR $\left(\mathrm{CDCl}_{3}, 600 \mathrm{MHz}\right) \delta 1.32$ (t, J $\left.7.14 \mathrm{~Hz}, 3 \mathrm{H}\right), 2.80$ (dd, J 5.34, $\left.18.18 \mathrm{~Hz}, 1 \mathrm{H}\right), 2.99$ (dd, J 9.78, $18.18 \mathrm{~Hz}, 1 \mathrm{H}), 3.72-3.74(\mathrm{~m}, 1 \mathrm{H}), 4.37-4.41(\mathrm{~m}, 2 \mathrm{H}), 4.53(\mathrm{~s}, 2 \mathrm{H}), 7.06(\mathrm{t}, J 8.40 \mathrm{~Hz}, 2 \mathrm{H}), 7.21-7.23(\mathrm{~m}, 2 \mathrm{H})$; ${ }^{13} \mathrm{C} \mathrm{NMR}\left(\mathrm{CDCl}_{3}, 150 \mathrm{MHz}\right) \delta 14.6,33.2,34.4,66.9,75.2,90.8,115.9,116.1,128.1,128.5,161.3,175.2,181.2$, 194.1; HRMS (ESI) Calcd. for $\mathrm{C}_{16} \mathrm{H}_{14} \mathrm{FNNaO}_{5}[\mathrm{M}+\mathrm{Na}]^{+}$: 342.0754; Found: 342.0748.

1-(4-Chlorophenyl)-3-(2-ethoxy-4-oxo-4,5-dihydrofuran-3-yl)pyrrolidine-2,5-dione (3e). White solid; 93\% yield; mp 171.2-172.5 ${ }^{\circ} \mathrm{C} .{ }^{1} \mathrm{H}$ NMR $\left(\mathrm{CDCl}_{3}, 600 \mathrm{MHz}\right) \delta 1.33(\mathrm{t}, J 7.08 \mathrm{~Hz}, 3 \mathrm{H}), 2.82$ (dd, J 5.46, 18.18 Hz, 1H), $3.01(\mathrm{dd}, J 9.78,18.12 \mathrm{~Hz}, 1 \mathrm{H}), 3.72-3.75(\mathrm{~m}, 1 \mathrm{H}), 4.37-4.41(\mathrm{~m}, 2 \mathrm{H}), 4.54(\mathrm{~s}, 2 \mathrm{H}), 7.20(\mathrm{~d}, J 8.52 \mathrm{~Hz}, 2 \mathrm{H}), 7.34(\mathrm{~d}$, J $8.58 \mathrm{~Hz}, 2 \mathrm{H}) ;{ }^{13} \mathrm{C} \mathrm{NMR}\left(\mathrm{CDCl}_{3}, 150 \mathrm{MHz}\right) \delta 14.6,33.3,34.4,66.9,75.2,90.7,127.9,129.2,130.7,134.3,174.9$, 176.1, 181.2, 194.0; HRMS (ESI) Calcd. for $\mathrm{C}_{16} \mathrm{H}_{14} \mathrm{NNaO}_{5}[\mathrm{M}+\mathrm{Na}]^{+}: 358.0458$; Found: 358.0453.

1-(4-Bromophenyl)-3-(2-ethoxy-4-oxo-4,5-dihydrofuran-3-yl)pyrrolidine-2,5-dione (3f). White solid; 92\% yield; mp 172.5-173.5 ${ }^{\circ} \mathrm{C} .{ }^{1} \mathrm{H}$ NMR $\left(\mathrm{CDCl}_{3}, 600 \mathrm{MHz}\right) \delta 1.35(\mathrm{t}, J 7.14 \mathrm{~Hz}, 3 \mathrm{H}), 2.85$ (dd, J 5.52, $\left.18.18 \mathrm{~Hz}, 1 \mathrm{H}\right), 3.01(\mathrm{dd}$, J 9.84, $18.18 \mathrm{~Hz}, 1 \mathrm{H}), 3.74(\mathrm{dd}, J 5.52,9.78 \mathrm{~Hz}, 1 \mathrm{H}), 4.40-4.43(\mathrm{~m}, 2 \mathrm{H}), 4.55(\mathrm{~s}, 2 \mathrm{H}), 7.15(\mathrm{~d}, J 8.58 \mathrm{~Hz}, 2 \mathrm{H}), 7.51$ $(d, J 8.64 \mathrm{~Hz}, 2 \mathrm{H}) ;{ }^{13} \mathrm{C} \mathrm{NMR}\left(\mathrm{CDCl}_{3}, 150 \mathrm{MHz}\right) \delta 14.0,32.7,33.7,66.3,74.6,90.0,121.7,127.5,130.5,131.6$, 174.2, 175.3, 180.5, 193.4; HRMS (ESI) Calcd. for $\mathrm{C}_{16} \mathrm{H}_{14} \mathrm{NNaO}_{5}[\mathrm{M}+\mathrm{Na}]^{+}$: 401.9953; Found: 401.9948.

3-(2-Ethoxy-4-oxo-4,5-dihydrofuran-3-yl)-1-(3-nitrophenyl)pyrrolidine-2,5-dione (3g). White solid; 85\% yield; $\mathrm{mp}$ 168.2-169.5 ${ }^{\circ} \mathrm{C} .{ }^{1} \mathrm{H}$ NMR $\left(\mathrm{CDCl}_{3}, 600 \mathrm{MHz}\right) \delta 1.39(\mathrm{t}, J 7.14 \mathrm{~Hz}, 3 \mathrm{H}), 2.92$ (dd, J 5.46, $\left.18.24 \mathrm{~Hz}, 1 \mathrm{H}\right), 3.09$ (dd, J 9.78, 18.24 Hz, 1H), 3.79-3.82 (m, 1H), 4.44-4.48 (m, 2H), $4.59(\mathrm{~s}, 2 \mathrm{H}), 7.59(\mathrm{t}, J 8.16 \mathrm{~Hz}, 1 \mathrm{H}), 7.68-7.69(\mathrm{~m}$, $1 \mathrm{H}), 8.18-8.21(\mathrm{~m}, 2 \mathrm{H}) ;{ }^{13} \mathrm{C} \mathrm{NMR}\left(\mathrm{CDCl}_{3}, 150 \mathrm{MHz}\right) \delta 13.9,32.6,33.6,66.3,74.5,89.7,121.1,122.4,129.1$, $131.8,132.4,147.6,173.6,174.9,180.4,193.2$; HRMS (ESI) Calcd. for $\mathrm{C}_{16} \mathrm{H}_{14} \mathrm{~N}_{2} \mathrm{NaO}_{7}[\mathrm{M}+\mathrm{Na}]^{+}$: 369.0699; Found: 369.0694.

3-(2-Ethoxy-4-oxo-4,5-dihydrofuran-3-yl)-1-(3-fluorophenyl)pyrrolidine-2,5-dione (3h). White solid; 87\% yield; $\mathrm{mp} 192.2-193.5^{\circ} \mathrm{C} .{ }^{1} \mathrm{H} \mathrm{NMR}\left(\mathrm{CDCl}_{3}, 600 \mathrm{MHz}\right) \delta 1.34(\mathrm{t}, J 7.08 \mathrm{~Hz}, 3 \mathrm{H}), 2.83(\mathrm{dd}, J 5.52,18.18 \mathrm{~Hz}, 1 \mathrm{H}), 3.01$ (dd, J 9.78, $18.12 \mathrm{~Hz}, 1 \mathrm{H}), 3.74-3.76(\mathrm{~m}, 1 \mathrm{H}), 4.39-4.42(\mathrm{~m}, 2 \mathrm{H}), 4.55(\mathrm{~s}, 2 \mathrm{H}), 7.02(\mathrm{t}, J 8.40 \mathrm{~Hz}, 2 \mathrm{H}), 7.07(\mathrm{~d}, J 8.04 \mathrm{~Hz}$, $1 \mathrm{H})$, 7.33-7.37 (m, 1H); ${ }^{13} \mathrm{C} \mathrm{NMR}\left(\mathrm{CDCl}_{3}, 150 \mathrm{MHz}\right) \delta 14.6,33.3,34.3,66.6,74.9,90.3,114.0,114.1,115.1$, 121.9, 129.8, 133.4, 163.2, 174.7, 181.2, 193.7; HRMS (ESI) Calcd. for $\mathrm{C}_{16} \mathrm{H}_{14} \mathrm{NNaO}_{5}[\mathrm{M}+\mathrm{Na}]^{+}: 342.0754$; Found: 342.0748.

3-(2-Ethoxy-4-oxo-4,5-dihydrofuran-3-yl)-1-(o-tolyl)pyrrolidine-2,5-dione (3i). White solid; 91\% yield; mp 141.0-142.5 ${ }^{\circ} \mathrm{C} .{ }^{1} \mathrm{H} \mathrm{NMR}\left(\mathrm{CDCl}_{3}, 600 \mathrm{MHz}\right) \delta 1.33(\mathrm{t}, J 7.08 \mathrm{~Hz}, 3 \mathrm{H}), 2.17(\mathrm{~s}, 3 \mathrm{H}), 2.91(\mathrm{dd}, J$ 6.30, $18.06 \mathrm{~Hz}, 1 \mathrm{H})$, $3.06(\mathrm{dd}, J$ 9.84, $18.18 \mathrm{~Hz}, 1 \mathrm{H}), 3.77-3.81(\mathrm{~m}, 1 \mathrm{H}), 4.37-4.41(\mathrm{~m}, 2 \mathrm{H}), 4.53(\mathrm{~s}, 2 \mathrm{H}), 6.98(\mathrm{~d}, J 7.68 \mathrm{~Hz}, 1 \mathrm{H}), 7.17-$ $7.24(\mathrm{~m}, 3 \mathrm{H}) ;{ }^{13} \mathrm{C} \mathrm{NMR}\left(\mathrm{CDCl}_{3}, 150 \mathrm{MHz}\right) \delta 14.7,17.6,33.4,34.1,66.8,75.2,90.3,126.7,127.9,129.4,131.1$, 131.3, 136.4, 175.2, 176.2, 181.2, 194.1; HRMS (ESI) Calcd. for $\mathrm{C}_{17} \mathrm{H}_{17} \mathrm{NNaO}_{5}[\mathrm{M}+\mathrm{Na}]^{+}$: 338.1004; Found: 338.0999.

3-(2-Ethoxy-4-oxo-4,5-dihydrofuran-3-yl)-1-methylpyrrolidine-2,5-dione (3j). White solid; 96\% yield; mp 103.5-104.5 ${ }^{\circ} \mathrm{C} .{ }^{1} \mathrm{H} \mathrm{NMR}\left(\mathrm{CDCl}_{3}, 600 \mathrm{MHz}\right) \delta 1.35(\mathrm{t}, J 7.14 \mathrm{~Hz}, 3 \mathrm{H}), 2.71(\mathrm{dd}, J 5.64,17.94 \mathrm{~Hz}, 1 \mathrm{H}), 2.83(\mathrm{dd}, J$ 9.54, $17.94 \mathrm{~Hz}, 1 \mathrm{H}), 2.96(\mathrm{~s}, 3 \mathrm{H}), 3.61(\mathrm{dd}, J 5.64,9.48 \mathrm{~Hz}, 1 \mathrm{H}), 4.39-4.43(\mathrm{~m}, 2 \mathrm{H}), 4.54(\mathrm{~s}, 2 \mathrm{H}) ;{ }^{13} \mathrm{C} \mathrm{NMR}\left(\mathrm{CDCl}_{3}\right.$, $150 \mathrm{MHz}$ ) $\delta 14.6,25.0,33.1,34.0,66.8,75.0,90.4,176.1,177.3,181.2$, 194.0; HRMS (ESI) Calcd. for $\mathrm{C}_{11} \mathrm{H}_{13} \mathrm{NNaO}_{5}[\mathrm{M}+\mathrm{Na}]^{+}: 262.0691$; Found: 262.0686.

1-Cyclohexyl-3-(2-ethoxy-4-oxo-4,5-dihydrofuran-3-yl)pyrrolidine-2,5-dione (3k). White solid; 92\% yield; mp 115.2-116.5 ${ }^{\circ} \mathrm{C} .{ }^{1} \mathrm{H} \mathrm{NMR}\left(\mathrm{CDCl}_{3}, 600 \mathrm{MHz}\right) \delta 1.13-1.27(\mathrm{~m}, 4 \mathrm{H}), 1.34(\mathrm{t}, \mathrm{J} 7.08 \mathrm{~Hz}, 3 \mathrm{H}), 1.55-1.59(\mathrm{~m}, 2 \mathrm{H}), 1.75(\mathrm{~d}$, J $12.72 \mathrm{~Hz}, 2 \mathrm{H}), 2.06-2.12(\mathrm{~m}, 2 \mathrm{H}), 2.60(\mathrm{dd}, J 5.58,18.00 \mathrm{~Hz}, 1 \mathrm{H}), 2.81(\mathrm{dd}, J$ 9.72, $17.94 \mathrm{~Hz}, 1 \mathrm{H}), 3.52-3.54(\mathrm{~m}$, $1 \mathrm{H}), 3.93(\mathrm{t}, J 12.30 \mathrm{~Hz}, 1 \mathrm{H}), 4.38-4.42(\mathrm{~m}, 2 \mathrm{H}), 4.53(\mathrm{~s}, 2 \mathrm{H}) ;{ }^{13} \mathrm{C} \mathrm{NMR}\left(\mathrm{CDCl}_{3}, 150 \mathrm{MHz}\right) \delta$ 14.6, 25.0, 25.8, 28.6, 
32.6, 34.1, 52.0, 66.7, 75.1, 91.0, 176.3, 177.2, 181.1, 194.1; HRMS (ESI) Calcd. for $\mathrm{C}_{16} \mathrm{H}_{21} \mathrm{NNaO}_{5}[\mathrm{M}+\mathrm{Na}]^{+}$: 330.1317; Found: 330.1312.

1-Benzyl-3-(2-ethoxy-4-oxo-4,5-dihydrofuran-3-yl)pyrrolidine-2,5-dione (3l). White solid; $90 \%$ yield; $\mathrm{mp}$ 118.0-119. $5^{\circ} \mathrm{C} .{ }^{1} \mathrm{H}$ NMR $\left(\mathrm{CDCl}_{3}, 600 \mathrm{MHz}\right) \delta 1.20(\mathrm{t}, J 7.14 \mathrm{~Hz}, 3 \mathrm{H}), 2.68(\mathrm{dd}, J 5.70,18.06 \mathrm{~Hz}, 1 \mathrm{H}), 2.85$ (dd, J 9.66, $18.06 \mathrm{~Hz}, 1 \mathrm{H}), 3.61-3.63(\mathrm{~m}, 1 \mathrm{H}), 4.27-4.29(\mathrm{~m}, 2 \mathrm{H}), 4.52(\mathrm{~s}, 2 \mathrm{H}), 4.58-4.64(\mathrm{~m}, 2 \mathrm{H}), 7.17(\mathrm{t}, J 7.38 \mathrm{~Hz}, 1 \mathrm{H})$, 7.22 (t, J $7.62 \mathrm{~Hz}, 2 \mathrm{H}), 7.31$ (d, J 7.32 Hz, 2H); ${ }^{13} \mathrm{C} \mathrm{NMR}\left(\mathrm{CDCl}_{3}, 150 \mathrm{MHz}\right) \delta$ 13.7, 32.1, 33.3, 41.7, 65.9, 74.4, 89.6, 126.8, 127.6, 127.7, 134.9, 174.9, 176.0, 180.2, 193.1; HRMS (ESI) Calcd. for $\mathrm{C}_{17} \mathrm{H}_{17} \mathrm{NNaO}_{5}[\mathrm{M}+\mathrm{Na}]^{+}$: 338.1004; Found: 338.0999.

1-Benzhydryl-3-(2-ethoxy-4-oxo-4,5-dihydrofuran-3-yl)pyrrolidine-2,5-dione (3m). White solid; $88 \%$ yield; $\mathrm{mp}$ 120.5-121.5 ${ }^{\circ} \mathrm{C} .{ }^{1} \mathrm{H}$ NMR $\left(\mathrm{CDCl}_{3}, 600 \mathrm{MHz}\right) \delta 1.23(\mathrm{t}, J 7.08 \mathrm{~Hz}, 3 \mathrm{H}), 2.74(\mathrm{dd}, J 6.00,18.12 \mathrm{~Hz}, 1 \mathrm{H}), 2.84(\mathrm{dd}, J$ 9.78, 18.12 Hz, 1H), 3.58-3.61 (m, 1H), 4.28-4.32 (m, 2H), $4.51(\mathrm{~s}, 2 \mathrm{H}), 6.51(\mathrm{~s}, 1 \mathrm{H}), 7.18-7.19(\mathrm{~m}, 2 \mathrm{H}), 7.20-7.25$ $(\mathrm{m}, 5 \mathrm{H}), 7.28(\mathrm{t}, J 7.74 \mathrm{~Hz}, 3 \mathrm{H}) ;{ }^{13} \mathrm{C} \mathrm{NMR}\left(\mathrm{CDCl}_{3}, 150 \mathrm{MHz}\right) \delta 14.6,32.9,34.0,58.6,66.8,75.2,90.6,127.6,128.3$, 128.7, 137.7, 175.6, 176.8, 181.2, 194.1; HRMS (ESI) Calcd. for $\mathrm{C}_{23} \mathrm{H}_{21} \mathrm{NNaO}_{5}[\mathrm{M}+\mathrm{Na}]^{+}$: 414.1317; Found: 414.1312.

3-(2-Ethoxy-4-oxo-4,5-dihydrofuran-3-yl)-1-(naphthalen-1-yl)pyrrolidine-2,5-dione (3n). White solid; 92\% yield; mp 122.5-123.5 ${ }^{\circ} \mathrm{C} .{ }^{1} \mathrm{H}$ NMR ( $\left.\mathrm{CDCl}_{3}, 600 \mathrm{MHz}\right) \delta 1.26$ (t, J $\left.7.08 \mathrm{~Hz}, 3 \mathrm{H}\right), 2.91$ (dd, J 5.40, $\left.18.24 \mathrm{~Hz}, 1 \mathrm{H}\right)$, $3.17(\mathrm{dd}, J$ J 9.9, $18.24 \mathrm{~Hz}, 1 \mathrm{H}), 3.82-3.83(\mathrm{~m}, 1 \mathrm{H}), 4.29-4.32(\mathrm{~m}, 2 \mathrm{H}), 4.52(\mathrm{~s}, 2 \mathrm{H}), 7.21(\mathrm{~d}, J 7.20 \mathrm{~Hz}, 1 \mathrm{H}), 7.37-$ $7.48(\mathrm{~m}, 3 \mathrm{H}), 7.79-7.83(\mathrm{~m}, 2 \mathrm{H}), 7.90(\mathrm{~d}, J 8.40 \mathrm{~Hz}, 1 \mathrm{H}) ;{ }^{13} \mathrm{C} \mathrm{NMR}\left(\mathrm{CDCl}_{3}, 150 \mathrm{MHz}\right) \delta 14.6,33.7,34.5,66.7,75.2$, 90.6, 122.0, 122.9, 125.2, 126.1, 126.6, 127.2, 128.3, 129.5, 129.9, 134.3, 175.7, 176.8, 181.3, 194.4; HRMS (ESI) Calcd. for $\mathrm{C}_{20} \mathrm{H}_{17} \mathrm{NNaO}_{5}[\mathrm{M}+\mathrm{Na}]^{+}$: 374.1004; Found: 374.0999.

3-(2-Methoxy-4-oxo-4,5-dihydrofuran-3-yl)-1-phenylpyrrolidine-2,5-dione (3o). White solid; $94 \%$ yield; $\mathrm{mp}$ 88.7-89.8 ${ }^{\circ} \mathrm{C} .{ }^{1} \mathrm{H}$ NMR $\left(\mathrm{CDCl}_{3}, 600 \mathrm{MHz}\right) \delta 2.85$ (dd, J 5.64, $\left.18.12 \mathrm{~Hz}, 1 \mathrm{H}\right), 3.01$ (dd, J 9.84, $\left.18.12 \mathrm{~Hz}, 1 \mathrm{H}\right), 3.73-$ $3.76(\mathrm{~m}, 1 \mathrm{H}), 4.01(\mathrm{~s}, 3 \mathrm{H}), 4.56(\mathrm{~s}, 2 \mathrm{H}), 7.24(\mathrm{~d}, J 7.62 \mathrm{~Hz}, 2 \mathrm{H}), 7.31(\mathrm{t}, J 7.44 \mathrm{~Hz}, 1 \mathrm{H}), 7.39(\mathrm{t}, J 7.80 \mathrm{~Hz}, 2 \mathrm{H}) ;{ }^{13} \mathrm{C}$ NMR (CDCl, $150 \mathrm{MHz}) \delta 32.5,33.5,56.0,74.5,90.0,125.8,127.8,128.3,131.4,174.4,175.4,180.6,193.3$; HRMS (ESI) Calcd. for $\mathrm{C}_{15} \mathrm{H}_{13} \mathrm{NNaO}_{5}[\mathrm{M}+\mathrm{Na}]^{+}:$310.0691; Found: 310.0686.

\section{Acknowledgements}

We appreciate the financial support from the National Natural Science Foundation of China (No. 21672031 and 81401535), Funds for Young Science and Technology Talent Cultivation Plan of Chongqing City (cstc2014kjrc-qnrc00004), Fundamental and Advanced Research Projects of Chongqing City (No. cstc2016jcyjA0267), Scientific and Technological Research Program of Chongqing Municipal Education Commission (No. KJ1600211) and Outstanding Young Scholars Project of Chongqing Medical University (No. 4101070059).

\section{Supplementary Material}

Supporting Information:

Spectra of all compounds, crystallographic data of $3 a$. 


\section{References}

1. Sakamoto, H. T.; Flausino, D.; Castellano, E. E.; Stark, C. B. W.; Gates, P. J.; Lopes, N. P. J. Nat. Prod. 2003, 66, 693-695.

http://dx.doi.org/10.1021/np020314v

2. Shin, S. S.; Byun, Y.; Lim, K. M.; Choi, J. K.; Lee, K.-W.; Moh, J. H.; Kim, J. K.; Jeong, Y. S.; Kim, J. Y.; Choi, Y. H.; Koh, H.-J.; Park, Y.-H.; Oh, Y. I.; Noh, M.-S.; Chung, S. J. Med. Chem. 2004, 47, 792-804. http://dx.doi.org/10.1021/im020545z

3. Pashkovskii, F. S.; Shchukina, E. M.; Gribovskii, M. G.; Lakhvich, F. A. Russ. J. Org. Chem. 2006, 42, 527540.

http://dx.doi.org/10.1134/S1070428006040087

4. Shamshina, J. L.; Snowden, T. S. Tetrahedron Lett. 2007, 48, 3767-3769. http://dx.doi.org/10.1016/j.tetlet.2007.03.166

5. Jerris, P. J.; Smith III, A. B. J. Org. Chem. 1981, 46, 577-585. http://dx.doi.org/10.1021/jo00316a018

6. Smith III, A. B.; Levenberg, P. A.; Jerris, P. J.; Scarborough Jr., R. M.; Wovkulich, P. M. J. Am. Chem. Soc. 1981, 103, 1501-1513.

http://dx.doi.org/10.1021/ja00396a034

7. Antonioletti, R.; Bonadies, F.; Prencipe, T.; Scettri, A. J. Chem. Soc., Chem. Commun. 1988, 850. http://dx.doi.org/10.1039/c39880000850

8. Winkler, J. D.; Oh, K.; Asselin, S. M. Org. Lett. 2005, 7, 387-389. http://dx.doi.org/10.1021/ol047810q

9. Liu, Y.; Liu, M.; Guo, S.; Tu, H.; Zhou, Y.; Gao, H. Org. Lett. 2006, 8, 3445-3448. http://dx.doi.org/10.1021/ol061059z

10. Kirsch, S. F.; Binder, J. T.; Liébert, C.; Menz, H. Angew. Chem. Int. Ed. 2006, 45, 5878-5880. http://dx.doi.org/10.1002/anie.200601836

11. Silva, F.; Reiter, M.; Mills-Webb, R.; Sawicki, M.; Klär, D.; Bensel, N.;Wagner, A.; Gouverneur, V. J. Org. Chem. 2006, 71, 8390-8394.

http://dx.doi.org/10.1021/jo061292a

12. Crone, B.; Kirsch, S. F. J. Org. Chem. 2007, 72, 5435-5438.

http://dx.doi.org/10.1021/jo070695n

13. Marson, C. M.; Edaan, E.; Morrell, J. M.; Coles, S. J.; Hursthouse, M. B.; Davies, D. T. Chem. Commun. 2007, 2494-2496.

http://dx.doi.org/10.1039/b701548h

14. Bunnelle, E. M.; Smith, C. R.; Lee, S. K.; Singaram, S. W.; Rhodes, A. J.; Sarpong, R. Tetrahedron. 2008, 64, 7008-7014.

http://dx.doi.org/10.1016/i.tet.2008.02.103

15. Egi, M.; Azechi, K.; Saneto, M.; Shimizu, K.; Akai, S. J. Org. Chem. 2010, 75, 2123-2126. http://dx.doi.org/10.1021/jo100048j

16. Poonoth, M; Krause, N. J. Org. Chem. 2011, 76, 1934-1936.

http://dx.doi.org/10.1021/jo102416e

17. Izquierdo, J.; Pericàs, M. A. ACS Catal. 2016, 6, 348-356. 
http://dx.doi.org/10.1021/acscatal.5b02121

18. Pellissier, H. Adv. Synth. Catal. 2016, 358, 2194-2259.

http://dx.doi.org/10.1002/adsc.201600462

19. Zhao, K.; Zhi, Y.; Shu, T.; Valkonen, A.; Rissanen, Kari P.; Enders, D. Angew. Chem. Int. Ed. 2016, 55, 12104-12108.

http://dx.doi.org/10.1002/anie.201606947

20. Diez, E. S.; Vesga, D. L.; Reyes, E.; Uria, U.; Carrillo, L.; Vicario, J. L. Org. Lett. 2016, 18, 1270-1273. http://dx.doi.org/10.1021/acs.orglett.6b00173

21. Dou, X.-W.; Han, X.-Y.; Lu, Y.-X. Chem.-Eur.J. 2012, 18, 85-89. http://dx.doi.org/10.1002/chem.201102796

22. Yan, Y.-Y.; Lu, R.-J.; Wang, J.-J.; Xuan, Y.-N.; Yan, M. Tetrahedron 2012, 68, 6123-6130. http://dx.doi.org/10.1016/i.tet.2012.05.082

23. John, J.; Hopf, H. Eur. J. Org. Chem. 2013, 841-845. http://dx.doi.org/10.1002/ejoc.201201253

24. Luo, N.-H.; Sun, X.; Yan, Y.-Y.; Nie, S.-Z.; Yan, M. Tetrahedron: Asymmetry 2011, 22, 1536-1541. http://dx.doi.org/10.1016/j.tetasy.2011.08.022

25. John, J.; Târcoveanu, E.; Jones, P. G.; Hopf, H. Beilstein J. Org. Chem. 2014, 10, 1462-1470. http://dx.doi.org/10.3762/bjoc.10.150

26. Wang, X.-B.; Li, T.-Z.; Sha, F.; Wu, X.-Y. Eur. J. Org. Chem. 2014, 739-744. http://dx.doi.org/10.1002/ejoc.201301350

27. Zou, C. C.; Zeng, C. K.; Liu, Z.; Lu, M.; Sun, X. H.; Ye, J. X. Angew. Chem. Int. Ed. 2016, 55, 14257-14261. http://dx.doi.org/10.1002/anie.201605790

28. Morita, T.; Akita, M.; Satoh, T.; Kakiuchi,F.; Miura, M. Org. Lett. 2016, 18, 4598-4601. http://dx.doi.org/10.1021/acs.orglett.6b02244

29. Kalia, D.; Malekar, P. V.; Parthasarathy, M. Angew. Chem. Int. Ed. 2016, 55, 1432-1435. http://dx.doi.org/10.1002/anie.201508118

30. Qiu, S.; Lee, R.; Zhu, B.; Coote, M.L.; Zhao, X. W.; Jiang, Z. Y. J. Org. Chem. 2016, 81, 8061-8069. http://dx.doi.org/10.1021/acs.joc.6b01451

31. Yoshimura1, A.; Nguyen, K. C.; Rohde, G. T.; Saito, A.; Yusubov, M. S.; Zhdankin, V. V. Adv.Synth.Catal. 2016, 358, 2340-2344.

http://dx.doi.org/10.1002/adsc.201600331

32. Zhou, J.; Wang, Q.-L.; Peng, L.; Tian, F.; Xu, X.-Y.; Wang, L.-X. Chem. Commun. 2014, 50, 14601-14605. http://dx.doi.org/10.1039/c4cc05207b

33. Zhou, J.; Jia, L.-N.; Wang, Q.-L.; Peng, L.; Tian, F.; Xu, X.-Y.; Wang, L.-X. Tetrahedron 2014, 70, 8665-8671. http://dx.doi.org/10.1016/j.tet.2014.09.040

34. Zhou, J.; Jia, L.-N.; Peng, L.; Wang, Q.-L.; Tian, F.; Xu, X.-Y.; Wang, L.-X. Tetrahedron 2014, 70, 3478-3484. http://dx.doi.org/10.1016/j.tet.2014.03.065

35. Wang, Q.-L.; Cai, T.; Zhou, J.; Tian, F.; Xu, X.-Y.; Wang, L.-X. Chem. Commun. 2015, 51, 10726-10729. http://dx.doi.org/10.1039/C5CC03793J

36. Guo, Y.-L.; Jia, L.-N.; Peng, L.; Qi, L.-W.; Zhou, J.; Tian, F.; Xu, X.-Y.; Wang, L.-X. RSC Adv. 2013, 3, 1697316976.

http://dx.doi.org/10.1039/c3ra43344g 
37. Rios, R.; Vesely, J.; Sundén, H.; Ibrahem, I.; Zhao, G. L.; Córdova, A. Tetrahedron Lett. 2007, 48, 58355839.

http://dx.doi.org/10.1016/i.tetlet.2007.06.070

38. The CCDC number is 1476572 .

39. Cava, M. P.; Deana, A. A.; Muth, K.; Mitchell, M. J. Org. Synth. 1961, 41, 93-93. http://dx.doi.org/10.1002/0471264180.os041.24 